\title{
OPTIMAL ALLOCATION OF ACTIVE SPARES IN SERIES SYSTEMS AND COMPARISON OF COMPONENT AND SYSTEM REDUNDANCIES
}

\author{
NEERAJ MISRA, ${ }^{*}$ \\ ISHWARI D. DHARIYAL * AND \\ NITIN GUPTA, ${ }^{* *}$ Indian Institute of Technology Kanpur
}

\begin{abstract}
We consider the problem of allocating $k$ active spares to $n$ components of a series system in order to optimize its lifetime. Under the hypotheses that lifetimes of $n$ components are identically distributed with distribution function $F(\cdot)$, lifetimes of $k$ spares are identically distributed with distribution function $G(\cdot)$, lifetimes of components and spares are independently distributed, and that $\ln (G(x)) / \ln (F(x))$ is increasing in $x$, we show that the strategy of balanced allocation of spares optimizes the failure rate function of the system. Furthermore, under the hypotheses that lifetimes of $n$ components are stochastically ordered, lifetimes of $k$ spares are identically distributed, and that lifetimes of components and spares are independently distributed, we show that the strategy of balanced allocation of spares is superior to the strategy of allocating a larger number of components to stronger components. For coherent systems consisting of $n$ identical components with $n$ identical redundant (spare) components, we compare strategies of component and system redundancies under the criteria of reversed failure rate and likelihood ratio orderings. When spares and original components do not necessarily match in their life distributions, we provide a sufficient condition, on the structure of the coherent system, for the strategy of component redundancy to be superior to the strategy of system redundancy under reversed failure rate ordering. As a consequence, we show that, for $r$-out-of- $n$ systems, the strategy of component redundancy is superior to the strategy of system redundancy under the criterion of reversed failure rate ordering. When spares and original components match in their life distributions, we provide a necessary and sufficient condition, on the structure of the coherent system, for the strategy of component redundancy to be superior to the strategy of system redundancy under the likelihood ratio ordering. As a consequence, we show that, for $r$-out-of- $n$ systems, with spares and original components matching in their life distributions, the strategy of component redundancy is superior to the strategy of system redundancy under the likelihood ratio ordering.
\end{abstract}

Keywords: Active redundancy; system redundancy; component redundancy; coherent system; stochastic ordering; Schur concave; Schur convex; majorization; $r$-out-of- $n$ system

2000 Mathematics Subject Classification: Primary 90B25

Secondary 60E15; 60K10

\footnotetext{
Received 19 September 2008; revision received 7 December 2008.

* Postal address: Department of Mathematics and Statistics, Indian Institute of Technology Kanpur, Kanpur, 208016, India.

** Email address: guptan@iitk.ac.in
} 


\section{Introduction}

In many situations, the performance of a coherent system can be enhanced by attaching spare (or redundant) components to its components. The problem of optimally allocating redundant components to the components of a coherent system to optimize its reliability or some other system performance characteristic is of considerable interest in reliability engineering, and often it leads to interesting theoretical results in probability. Two types of commonly used redundancies are active (or parallel) redundancy and standby redundancy. In active redundancy, spares are put in parallel to components of the system and they start functioning at the same time as original components. In standby redundancy, spares are put in standby and they start functioning only after the failure of original components.

Boland et al. (1988), Shaked and Shanthikumar (1992), and Singh and Singh (1997a) considered the problem of optimally distributing $k$ active spares to $n$ components, $C_{1}, \ldots, C_{n}$, of a series system. Suppose that the lifetimes of $n$ components are independent with a common distribution function $F(\cdot)$ and that those of $k$ available spares are also independent and have a common distribution function $G(\cdot)$. Furthermore, assume that the lifetimes of components and spares are independently distributed. Let $S=\{0,1, \ldots, k\}$ and $\mathbb{K}=\left\{\boldsymbol{L}=\left(l_{1}, \ldots, l_{n}\right): \boldsymbol{L} \in\right.$ $S^{n}$ and $\left.\sum_{i=1}^{n} l_{i}=k\right\}$, where $S^{n}$ denotes the product space $S \times \cdots \times S$. Let $T_{S}(\boldsymbol{k})$ denote the lifetime of the series system when $k_{i}$ spares are put in parallel to $C_{i}, i=1, \ldots, n$, where $\boldsymbol{k}=\left(k_{1}, \ldots, k_{n}\right) \in \mathbb{K}$. Boland et al. (1988) proved that the survival function of $T_{s}(\boldsymbol{k})$ is a Schur-concave function of $\boldsymbol{k}$ on $\mathbb{K}$ (see Definition 1.1(b), below). As a consequence, it follows that allocating the spares equally among $n$ components is optimal under the criterion of usual stochastic ordering. In fact, they proved this result for $r$-out-of- $n$ systems. Under the assumption that $F \equiv G$, Shaked and Shanthikumar (1992) independently obtained the above result for series systems. Apart from the survival function, the failure rate function is another important system performance characteristic which is widely used in reliability engineering. Singh and Singh (1997a) proved that if the lifetimes of components and spares are independent and identically distributed, then the failure rate function of $T_{S}(\boldsymbol{k})$ is a Schur-convex function of $\boldsymbol{k}$ on $\mathbb{K}$. Consequently, it follows that the balanced allocation of spares to $n$ components of the series system optimizes the failure rate function of the system. Since the failure rate ordering is stronger than the usual stochastic ordering, when $F \equiv G$, results obtained in Singh and Singh (1997a) are stronger than those obtained in Boland et al. (1988).

In Section 2 we show that if $\ln (G(x)) / \ln (F(x))$ is an increasing function of $x$ then the failure rate function of $T_{S}(\boldsymbol{k})$ is a Schur-convex function of $\boldsymbol{k}$ on $\mathbb{K}$-strengthening the result of Singh and Singh (1997a). Furthermore, when the lifetimes of $n$ components are independent with stochastically ordered distribution functions $F_{1}, \ldots, F_{n}\left(F_{1}(x) \leq \cdots \leq F_{n}(x)\right.$ for all $\left.x\right)$ and the spares are independent and identically distributed with distribution function $G(\cdot)$, we prove that the survival function of $T_{S}(\boldsymbol{k})$ is a Schur-concave function of $\boldsymbol{k}$ on $\mathbb{K}_{R}=\{\boldsymbol{k}: \boldsymbol{k} \in$ $\left.\mathbb{K}, k_{1} \geq k_{2} \geq \cdots \geq k_{n}\right\}$. Therefore, under these generalized assumptions, the strategy of balanced allocation is superior to the strategy of allocating a larger number of components to stronger components. A result derived in Boland et al. (1988) can be obtained as a particular case of this result.

In reliability engineering, it is well known that a coherent system where active spare allocation is made at the component level has a lifetime stochastically larger than that of the system where active spare allocation is made at the system level. However, as shown in Boland and El-Neweihi (1995), the above result is not true with respect to the failure rate ordering if lifetimes of components and corresponding spares are not identically distributed. They conjectured that, in general, for $r$-out-of- $n$ systems, the component level active redundancy is 
better than the system level active redundancy with respect to the failure rate ordering provided that the lifetimes of components and spares are independent and identically distributed. Singh and Singh (1997b) resolved the conjecture of Boland and El-Neweihi (1995) and proved a stronger result: for $r$-out-of- $n$ systems consisting of components and spares having independent and identically distributed lifetimes, the component level redundancy is better than the system level redundancy with respect to likelihood ratio ordering.

In Section 3, under the assumption of independence of lifetimes of components and nonmatching spares, a sufficient condition on the structure function of the coherent system for the component level redundancy to be better than the system level redundancy with respect to the reversed failure rate ordering is derived. Consequently, it follows that, for $r$-out-of- $n$ systems with possibly nonmatching spares (i.e. general $F$ and $G$ ), the component level redundancy is better than the system level redundancy under the criterion of the reversed failure rate ordering. Note that, for $r$-out-of- $n$ systems, such a result does not hold with respect to the failure rate ordering. Under the additional assumption of matching spares, a necessary and sufficient condition on the structure function of the coherent system for the component level redundancy to be better than the system level redundancy under the criterion of likelihood ratio ordering is given. As a corollary to this, we obtain the result proved in Singh and Singh (1997b).

All the random variables considered in this paper are assumed to have the Lebesgue probability density functions and the support $[0, \infty)=\mathbb{R}_{+}$. Moreover, for a set $A$ and a positive integer $m, A^{m}$ denotes the product set $A \times \cdots \times A$, and $\wedge\left\{x_{1}, \ldots, x_{n}\right\}$ and $\vee\left\{x_{1}, \ldots, x_{n}\right\}$ respectively denote the smallest and the largest real numbers of $x_{1}, \ldots, x_{n}$. Throughout the paper, increasing and decreasing are used for nondecreasing and nonincreasing, respectively.

In order to make the presentation self-contained, we reproduce the following definitions from the literature (see Marshall and Olkin (1979, pp. 7 and 54), Barlow and Proschan (1975), and Shaked and Shanthikumar (2007, p. 2)).

Definition 1.1. (Majorization.) Let $\mathbb{R}^{n}$ denote the $n$-dimensional Euclidean space, and let $\mathbb{A} \subseteq \mathbb{R}^{n}$

(a) A point $\boldsymbol{x} \in \mathbb{A}$ is said to be majorized by another point $\boldsymbol{y} \in \mathbb{A}$ (written as $\boldsymbol{x} \leq_{\mathrm{m}} \boldsymbol{y}$ ) if

$$
\sum_{i=1}^{j} x_{[i]} \leq \sum_{i=1}^{j} y_{[i]}, \quad j=1,2, \ldots, n-1, \quad \text { and } \quad \sum_{i=1}^{n} x_{[i]}=\sum_{i=1}^{n} y_{[i]},
$$

where $x_{[1]} \geq \cdots \geq x_{[n]}$ and $y_{[1]} \geq \cdots \geq y_{[n]}$ denote the ranked values of $x_{1}, \ldots, x_{n}$ and $y_{1}, \ldots, y_{n}$, respectively.

(b) A function $\psi: \mathbb{A} \rightarrow \mathbb{R}$ is said to be Schur concave on $\mathbb{A}$ if

$$
\boldsymbol{x} \leq_{\mathrm{m}} \boldsymbol{y} \quad \Longrightarrow \quad \psi(\boldsymbol{x}) \geq \psi(\boldsymbol{y}) \quad \text { for } \boldsymbol{x}, \boldsymbol{y} \in \mathbb{A}
$$

and Schur convex on $\mathbb{A}$ if

$$
\boldsymbol{x} \leq_{\mathrm{m}} \boldsymbol{y} \quad \Longrightarrow \quad \psi(\boldsymbol{x}) \leq \psi(\boldsymbol{y}) \quad \text { for } \boldsymbol{x}, \boldsymbol{y} \in \mathbb{A} .
$$

Definition 1.2. Let $Z_{i}, i=1,2$, be two random variables with survival functions $\bar{H}_{i}(\cdot)$, distribution functions $H_{i}(\cdot)$, and probability density functions $h_{i}(\cdot)$, respectively. Then $Z_{1}$ is said to be smaller than $Z_{2}$ in the

(a) likelihood ratio order (written as $Z_{1} \leq_{\operatorname{lr}} Z_{2}$ ) if $h_{2}(x) / h_{1}(x)$ is an increasing function of $x$ on $(0, \infty)$ 
(b) failure rate order (written as $Z_{1} \leq$ fr $Z_{2}$ ) if $\bar{H}_{2}(x) / \bar{H}_{1}(x)$ is an increasing function of $x$ on $(0, \infty)$;

(c) reversed failure rate order (written as $Z_{1} \leq_{\mathrm{rfr}} Z_{2}$ ) if $H_{2}(x) / H_{1}(x)$ is an increasing function of $x$ on $(0, \infty)$;

(d) usual stochastic order (written as $Z_{1} \leq_{\text {st }} Z_{2}$ ) if $\bar{H}_{1}(x) \leq \bar{H}_{2}(x)$ for every $x \in \mathbb{R}$.

It is well known that (see, for example, Shaked and Shanthikumar (2007))

(i) $Z_{1} \leq_{\text {lr }} Z_{2} \Longrightarrow Z_{1} \leq_{\text {fr }} Z_{2} \Longrightarrow Z_{1} \leq_{\text {st }} Z_{2}$; and

(ii) $Z_{1} \leq$ lr $Z_{2} \Longrightarrow Z_{1} \leq_{\text {rfr }} Z_{2} \Longrightarrow Z_{1} \leq_{\text {st }} Z_{2}$.

\section{Optimal allocation of active spares in series systems}

Consider a series system $\phi_{S}$, consisting of $n$ components $C_{1}, C_{2}, \ldots, C_{n}$ having independent random lifetimes $X_{1}, X_{2}, \ldots, X_{n}$, respectively. Let $\tau(X)=\wedge\left\{X_{1}, \ldots, X_{n}\right\}$ denote the lifetime of $\phi_{s}$. Let $R_{1}, \ldots, R_{k}$ be $k$ active spares having independent and identically distributed random lifetimes $Y_{1}, \ldots, Y_{k}$, respectively. Consider the problem of optimally allocating these $k$ spares to $n$ components in parallel (active redundancy). Any element of the set

$$
\mathbb{K}=\left\{\boldsymbol{L}=\left(l_{1}, \ldots, l_{n}\right): l_{i}=0,1, \ldots, k, i=1, \ldots, n, \sum_{i=1}^{n} l_{i}=k\right\}
$$

is said to be an allocation. Let $T_{s}(\boldsymbol{k}), \boldsymbol{k} \in \mathbb{K}$, denote the lifetime of system $S_{\boldsymbol{k}}$ obtained from the series system $\phi_{s}$ by putting $k_{i}$ components in parallel to $C_{i}, i=1, \ldots, n$. Then the survival, failure rate, and the reversed failure rate functions of $T_{S}(\boldsymbol{k})$ are given by

$$
\begin{aligned}
\bar{F}_{s, \boldsymbol{k}}(x) & =\mathrm{P}\left(\wedge\left\{\vee\left\{X_{1}, Y_{1}, \ldots, Y_{k_{1}}\right\}, \ldots, \vee\left\{X_{n}, Y_{\sum_{i=1}^{n-1} k_{i}+1}, \ldots, Y_{\sum_{i=1}^{n} k_{i}}\right\}\right\}>x\right) \\
& =\prod_{i=1}^{n}\left(1-F_{i}(x) G^{k_{i}}(x)\right), \quad x \in \mathbb{R}_{+}, \\
r_{s, \boldsymbol{k}}(x) & =\sum_{i=1}^{n}\left(\frac{\left(\tilde{r}_{F_{i}}(x)+k_{i} \tilde{r}_{G}(x)\right) F_{i}(x) G^{k_{i}}(x)}{1-F_{i}(x) G^{k_{i}}(x)}\right), \quad x \in \mathbb{R}_{+},
\end{aligned}
$$

and

$$
\begin{aligned}
\tilde{r}_{s, \boldsymbol{k}}(x)= & \left(\frac{\prod_{i=1}^{n}\left(1-F_{i}(x) G^{k_{i}}(x)\right)}{1-\prod_{i=1}^{n}\left(1-F_{i}(x) G^{k_{i}}(x)\right)}\right) \\
& \times\left(\sum_{i=1}^{n}\left(\frac{\left(\tilde{r}_{F_{i}}(x)+k_{i} \tilde{r}_{G}(x)\right) F_{i}(x) G^{k_{i}}(x)}{1-F_{i}(x) G^{k_{i}}(x)}\right)\right), \quad x \in \mathbb{R}_{+},
\end{aligned}
$$

respectively, where $F_{i}(\cdot)$ and $\tilde{r}_{F_{i}}(\cdot)$ denote the distribution functions and reversed failure rate functions, respectively, of $X_{i}, i=1,2, \ldots, n$, and $G(\cdot)$ and $\tilde{r}_{G}(\cdot)$ denote the common distribution function and the reversed failure rate function, respectively, of the $Y$ s.

Under the simplifying assumption $F_{i} \equiv F, i=1, \ldots, n$, Boland et al. (1988) proved that $T_{S}(\boldsymbol{k}) \leq_{\mathrm{st}} T_{S}\left(\boldsymbol{k}_{\mathbf{0}}\right)$ for all $\boldsymbol{k}, \boldsymbol{k}_{\mathbf{0}} \in \mathbb{K}$ such that $\boldsymbol{k}_{\mathbf{0}} \leq_{\mathrm{m}} \boldsymbol{k}$. In fact, they proved this result for more general $r$-out-of- $n$ systems. However, as shown in Example 2.1, below, it is not true that $T_{s}(\boldsymbol{k}) \leq_{\mathrm{lr}} T_{s}\left(\boldsymbol{k}_{\mathbf{0}}\right)$ for all $\boldsymbol{k}, \boldsymbol{k}_{\mathbf{0}} \in \mathbb{K}$ such that $\boldsymbol{k}_{\mathbf{0}} \leq_{\mathrm{m}} \boldsymbol{k}$, even for matching components 
and spares, i.e. even if $F \equiv G$. This indicates that an attempt at generalizing the result of Boland et al. (1988) towards likelihood ratio ordering will be futile.

Example 2.1. Consider the system $S_{\boldsymbol{k}}$ with $n=3, \boldsymbol{k}_{\mathbf{0}}=(4,8,8), \boldsymbol{k}_{\mathbf{1}}=(4,5,11)$, and $F_{1} \equiv F_{2} \equiv F_{3} \equiv F \equiv G$. Note that $\boldsymbol{k}_{\mathbf{0}} \leq_{\mathrm{m}} \boldsymbol{k}_{\mathbf{1}}$. The probability density function of $T_{s}(\boldsymbol{k})$ is given by

$$
\begin{aligned}
f_{s, \boldsymbol{k}}(x)= & \tilde{r}_{F}(x)\left(1-F^{k_{1}+1}(x)\right)\left(1-F^{k_{2}+1}(x)\right)\left(1-F^{k_{3}+1}(x)\right) \\
& \times\left(\frac{\left(k_{1}+1\right) F^{k_{1}+1}(x)}{1-F^{k_{1}+1}(x)}+\frac{\left(k_{2}+1\right) F^{k_{2}+1}(x)}{1-F^{k_{2}+1}(x)}+\frac{\left(k_{3}+1\right) F^{k_{3}+1}(x)}{1-F^{k_{3}+1}(x)}\right), \quad x \geq 0 .
\end{aligned}
$$

Therefore,

$$
\frac{f_{s, \boldsymbol{k}_{\mathbf{0}}}(x)}{f_{s, \boldsymbol{k}_{\mathbf{1}}}(x)}=\frac{\psi_{\boldsymbol{k}_{\mathbf{0}}}(F(x))}{\psi_{\boldsymbol{k}_{\mathbf{1}}}(F(x))}, \quad x \geq 0,
$$

where, for $0<p<1$ and $\boldsymbol{k} \in \mathbb{K}$,

$$
\begin{aligned}
\psi_{\boldsymbol{k}}(p)= & \left(k_{1}+1\right) p^{k_{1}+1}\left(1-p^{k_{2}+1}\right)\left(1-p^{k_{3}+1}\right) \\
& +\left(k_{2}+1\right)\left(1-p^{k_{1}+1}\right) p^{k_{2}+1}\left(1-p^{k_{3}+1}\right) \\
& +\left(k_{3}+1\right)\left(1-p^{k_{1}+1}\right)\left(1-p^{k_{2}+1}\right) p^{k_{3}+1} .
\end{aligned}
$$

Let $\psi(p)=\psi_{\boldsymbol{k}_{\mathbf{0}}}(p) / \psi_{\boldsymbol{k}_{\mathbf{1}}}(p), p \in(0,1)$. Since,

$$
\psi(0.1) \approx 0.8932, \quad \psi(0.5) \approx 0.7658, \quad \text { and } \quad \psi(0.9) \approx 1.1075
$$

it is clear that, while $\boldsymbol{k}_{\mathbf{0}} \leq_{\mathrm{m}} \boldsymbol{k}$, it is not true that $T_{S}(\boldsymbol{k}) \leq_{\operatorname{lr}} T_{S}\left(\boldsymbol{k}_{\mathbf{0}}\right)$.

For the system $S_{k}$ with identical components and matching spares, i.e. when $F \equiv G$, Singh and Singh (1997a) proved that $T_{S}(\boldsymbol{k}) \leq_{\mathrm{fr}} T_{S}\left(\boldsymbol{k}_{\mathbf{0}}\right)$ for all $\boldsymbol{k}, \boldsymbol{k}_{\mathbf{0}} \in \mathbb{K}$ such that $\boldsymbol{k}_{\mathbf{0}} \leq_{\mathrm{m}} \boldsymbol{k}$. Example 2.2 given below illustrates that this result may not hold when $F$ and $G$ are not the same. Thus, it will be worth investigating whether the result holds under some conditions on the distribution functions $F(\cdot)$ and $G(\cdot)$.

Example 2.2. Consider the system $S_{k}$ with $n=2, k=k_{1}+k_{2}=10, F_{1}(x)=F_{2}(x)=1-\mathrm{e}^{-x}$ for all $x \geq 0$, and $G(x)=1-\mathrm{e}^{-2 x}$ for all $x \geq 0$. Then the failure rate function given by (2.2) reduces to

$$
\begin{aligned}
r_{s,\left(k_{1}, k_{2}\right)=} & \frac{\mathrm{e}^{-x}\left(1-\mathrm{e}^{-2 x}\right)^{k_{1}}+2 k_{1} \mathrm{e}^{-2 x}\left(1-\mathrm{e}^{-x}\right)\left(1-\mathrm{e}^{-2 x}\right)^{k_{1}-1}}{1-\left(1-\mathrm{e}^{-x}\right)\left(1-\mathrm{e}^{-2 x}\right)^{k_{1}}} \\
& +\frac{\mathrm{e}^{-x}\left(1-\mathrm{e}^{-2 x}\right)^{k_{2}}+2 k_{2} \mathrm{e}^{-2 x}\left(1-\mathrm{e}^{-x}\right)\left(1-\mathrm{e}^{-2 x}\right)^{k_{2}-1}}{1-\left(1-\mathrm{e}^{-x}\right)\left(1-\mathrm{e}^{-2 x}\right)^{k_{2}}} .
\end{aligned}
$$

A simple calculation shows that

$$
r_{s,(5,5)}(x)-r_{s,(0,10)}(x) \approx \begin{cases}-0.9650 & \text { for } x=0.2, \\ +0.3036 & \text { for } x=1.2,\end{cases}
$$

which implies that $T_{S}(\boldsymbol{k}) \leq_{\mathrm{fr}} T_{S}\left(\boldsymbol{k}_{\mathbf{0}}\right)$ for all $\boldsymbol{k}, \boldsymbol{k}_{\mathbf{0}} \in \mathbb{K}$ such that $\boldsymbol{k}_{\mathbf{0}} \leq_{\mathrm{m}} \boldsymbol{k}$ does not hold in this case. 
This example gives motivation for finding conditions on $F(\cdot)$ and $G(\cdot)$ under which $T_{s}(\boldsymbol{k}) \leq_{\mathrm{fr}}$ $T_{s}\left(\boldsymbol{k}_{\mathbf{0}}\right)$ for all $\boldsymbol{k}, \boldsymbol{k}_{\mathbf{0}} \in \mathbb{K}$ satisfying $\boldsymbol{k}_{\mathbf{0}} \leq_{\mathrm{m}} \boldsymbol{k}$. A set of such conditions is given in Theorem 2.1, below. The following lemma, whose proof is available in Marshall and Olkin (1979, Proposition C.1) and Pečarić et al. (1992, Theorem 1.36), is required for proving the theorem.

Lemma 2.1. Let $g: I \rightarrow \mathbb{R}$ be a function defined on an interval $I \subset \mathbb{R}$.

(a) If $g(\cdot)$ is convex on I then $\phi(x)=\sum_{i=1}^{n} g\left(x_{i}\right)$ is Schur convex on $I^{n}$, where $\boldsymbol{x}=$ $\left(x_{1}, \ldots, x_{n}\right)$.

(b) Suppose that $g(\cdot)$ is continuous and midpoint convex on I, i.e. $g(\cdot)$ is continuous on I and $g((u+v) / 2) \leq(g(u)+g(v)) / 2$ whenever $u, v \in I$. Then $\phi(\boldsymbol{x})=\sum_{i=1}^{n} g\left(x_{i}\right)$ is Schur convex on $I^{n}$.

Theorem 2.1. Let $F_{i} \equiv F, i=1, \ldots, n$. If $\ln (G(x)) / \ln (F(x))$ is an increasing function of $x$ on $\mathbb{R}_{+}$then

$$
\boldsymbol{k}, \boldsymbol{k}_{\mathbf{0}} \in \mathbb{K} \text { such that } \boldsymbol{k}_{\mathbf{0}} \leq_{\mathrm{m}} \boldsymbol{k} \quad \Longrightarrow \quad r_{s, \boldsymbol{k}_{\mathbf{0}}}(x) \leq r_{s, \boldsymbol{k}}(x) \text { for all } x \geq 0,
$$

i.e. $r_{s, \boldsymbol{k}}(x)$ is a Schur-convex function of $\boldsymbol{k}$ on $\mathbb{R}_{+}^{n}$.

Proof. We have

$$
r_{s, k}(x)=\sum_{i=1}^{n} g_{1}\left(k_{i}\right)
$$

where

$$
g_{1}(y)=\frac{F(x) G^{y}(x)\left(\tilde{r}_{F}(x)+y \tilde{r}_{G}(x)\right)}{1-F(x) G^{y}(x)}, \quad y \geq 0 .
$$

Fix $x \geq 0$. Let $p=F(x), q=G(x), a=\tilde{r}_{F}(x), b=\tilde{r}_{G}(x)$, and $g(y)=g_{1}(y)+b y+a$, $y \geq 0$. Note that $g(y)$ and $g_{1}(y)$ are continuous functions of $y$ on $\mathbb{R}_{+}$. Under the hypothesis of the theorem, we will prove that $g(y)$ (and, therefore, $g_{1}(y)$ ) is a midpoint convex function of $y$ on $\mathbb{R}_{+}$. The result would then follow using Lemma 2.1(b). We have

$$
g(y)=\frac{a+b y}{1-p q^{y}}, \quad y \geq 0 .
$$

Fix $0 \leq u<v<\infty$, and consider

$$
\begin{aligned}
A(u, v) & =g(u)+g(v)-2 g\left(\frac{u+v}{2}\right) \\
& =\frac{a+b u}{1-p q^{u}}+\frac{a+b v}{1-p q^{v}}-\frac{2 a+b u+b v}{1-p q^{(u+v) / 2}} \\
& =\frac{p\left(q^{u / 2}-q^{v / 2}\right)}{1-p q^{(u+v) / 2}}\left(\frac{a+b u}{q^{-u / 2}-p q^{u / 2}}-\frac{a+b v}{q^{-v / 2}-p q^{v / 2}}\right) .
\end{aligned}
$$

We need to show that $A(u, v) \geq 0$. Since, for $0<q<1, q^{x}$ is a decreasing function of $x$ on $\mathbb{R}_{+}$, to prove that $A(u, v) \geq 0$, it is enough to show that

$$
\psi_{1}(t)=\frac{a+b t}{q^{-t / 2}-p q^{t / 2}}
$$


is a decreasing function of $t$ on $\mathbb{R}_{+}$. We have

$$
\psi_{1}^{\prime}(t)=\frac{\mathrm{d}}{\mathrm{d} t}\left(\psi_{1}(t)\right)=\frac{\psi_{2}(t)}{\left(q^{-t / 2}-p q^{t / 2}\right)^{2}},
$$

where

$$
\psi_{2}(t)=b\left(q^{-t / 2}-p q^{t / 2}\right)+\frac{\ln (q)}{2}(a+b t)\left(q^{-t / 2}+p q^{t / 2}\right) .
$$

Also,

$$
\psi_{2}^{\prime}(t)=-\frac{(\ln (q))^{2}}{4}(a+b t)\left(q^{-t / 2}-p q^{t / 2}\right) \leq 0 \quad \text { for all } t \in \mathbb{R}_{+}
$$

and

$$
\begin{aligned}
\psi_{2}(0) & =b(1-p)+\frac{\ln (q)}{2} a(1+p) \\
& =\tilde{r}_{G}(x)(1-F(x))+\frac{\ln (G(x))}{2} \tilde{r}_{F}(x)(1+F(x)) .
\end{aligned}
$$

Since $\ln (G(x)) / \ln (F(x))$ is an increasing function of $x$ on $\mathbb{R}_{+}$, we have

$$
\ln (G(x)) \tilde{r}_{F}(x) \leq \ln (F(x)) \tilde{r}_{G}(x) \quad \text { for all } x \in \mathbb{R}_{+} .
$$

Using (2.6) in (2.5), we obtain

$$
\begin{aligned}
\psi_{2}(0) & \leq \tilde{r}_{G}(x)(1-F(x))+\frac{\ln (F(x))}{2} \tilde{r}_{G}(x)(1+F(x)) \\
& =\tilde{r}_{G}(x) \Delta(F(x))
\end{aligned}
$$

where, for $0<p \leq 1$,

$$
\Delta(p)=\frac{\ln (p)}{2}(1+p)+1-p
$$

Observe that

$$
\Delta^{\prime}(p)=\frac{1}{2 p}-\frac{1}{2}+\frac{\ln (p)}{2}
$$

and

$$
\Delta^{\prime \prime}(p)=\frac{\mathrm{d}^{2}}{\mathrm{~d} p^{2}} \Delta(p)=-\frac{1-p}{2 p^{2}} \leq 0 \quad \text { for all } 0<p \leq 1 .
$$

Consequently, for every $p \in(0,1]$,

$$
\Delta^{\prime}(p) \geq \Delta^{\prime}(1)=0 \quad \text { and } \quad \Delta(p) \leq \Delta(1)=0 .
$$

Using (2.8) in (2.7), it follows that $\psi_{2}(0) \leq 0$ for every $x \geq 0$. Now using (2.4), we conclude that $\psi_{2}(t) \leq 0$ for every $t \in \mathbb{R}_{+}$and, therefore, $\psi_{1}(t)$ is a decreasing function of $t$ on $\mathbb{R}_{+}$.

Lemma 2.2. If $F_{i} \equiv F, i=1, \ldots, n$, and $\tilde{r}_{G}(x) / \tilde{r}_{F}(x)$ is an increasing function of $x$ on $\mathbb{R}_{+}$, then $\ln (G(x)) / \ln (F(x))$ is an increasing function of $x$ on $\mathbb{R}_{+}$. 
Proof. Fix $x \geq 0$, and let $A>x$. Applying Cauchy's mean value theorem to functions $\ln (G(\cdot))$ and $\ln (F(\cdot))$ on the interval $[0, A]$ we conclude that

$$
\frac{\ln (G(A))-\ln (G(x))}{\ln (F(A))-\ln (F(x))}=\frac{\tilde{r}_{G}(\xi)}{\tilde{r}_{F}(\xi)} \quad \text { for some } \xi \in(x, A) .
$$

Since $\tilde{r}_{G}(x) / \tilde{r}_{F}(x)$ is increasing on $\mathbb{R}_{+}$, we obtain

$$
\frac{\ln (G(A))-\ln (G(x))}{\ln (F(A))-\ln (F(x))} \geq \frac{\tilde{r}_{G}(x)}{\tilde{r}_{F}(x)} .
$$

Since $A>x$ is arbitrary, on taking $A \rightarrow \infty$ we obtain

$$
\frac{\ln (G(x))}{\ln (F(x))} \geq \frac{\tilde{r}_{G}(x)}{\tilde{r}_{F}(x)} \quad \text { for all } x \geq 0 \quad \Longrightarrow \quad \frac{\mathrm{d}}{\mathrm{d} x}\left[\frac{\ln (G(x))}{\ln (F(x))}\right] \geq 0 \quad \text { for all } x \geq 0,
$$

i.e. $\ln (G(x)) / \ln (F(x))$ is an increasing function of $x$ on $\mathbb{R}_{+}$.

Remark 2.1. (i) Kalashnikov and Rachev (1986) proposed a stochastic order based on the notion of relative ageing of two probability distributions. Let $X$ and $Y$ be two random variables with distribution functions $F(\cdot)$ and $G(\cdot)$, survival functions $\bar{F}(\cdot)$ and $\bar{G}(\cdot)$, failure rate functions $r_{F}(\cdot)$ and $r_{G}(\cdot)$, and reversed failure rate functions $\tilde{r}_{F}(\cdot)$ and $\tilde{r}_{G}(\cdot)$, respectively. According to Kalashnikov and Rachev (1986), the random variable $X$ is said to be ageing faster than the random variable $Y$ (written as $X<_{c} Y$ ) if the random variable $Z=-\ln (\bar{G}(X)$ ) has an increasing failure rate (IFR) distribution. If $r_{G}(\cdot) \neq 0$ then it is easy to see that $X<_{\mathrm{c}} Y$ if and only if $r_{F}(\cdot) / r_{G}(\cdot)$ is an increasing function. Sengupta and Deshpande (1994) further studied this concept of relative ageing and proposed two new models of relative ageing and studied their interrelations. Similar to the notion of relative ageing defined in Kalashnikov and Rachev (1986), another notion of relative ageing can be defined in terms of the reversed failure rate. We say that the random variable $X$ is ageing faster than the random variable $Y$ in the reversed failure rate ordering (written as $X<_{\mathrm{rfr}-\mathrm{c}} Y$ ) if the random variable $Z=-\ln (G(X)$ ) has an IFR distribution. If $\tilde{r}_{F}(\cdot) \neq 0$ then it is easy to see that $X<_{\mathrm{rfr}-\mathrm{c}} Y$ if and only if $\tilde{r}_{G}(\cdot) / \tilde{r}_{F}(\cdot)$ is an increasing function.

(ii) According to Theorem 2.1, if $\ln (G(x)) / \ln (F(x))$ is an increasing function of $x$ on $\mathbb{R}_{+}$then a balanced allocation of spares (approximately an equal number of spares with each component) results in the lowest failure rate function and, hence, is optimal with respect to the failure rate criterion.

(iii) From Lemma 2.2, it follows that if $F_{i} \equiv F, i=1, \ldots, n$, and $\tilde{r}_{G}(x) / \tilde{r}_{F}(x)$ is an increasing function of $x$ on $\mathbb{R}_{+}$then the conclusion of Theorem 2.1 holds.

Note that the condition of Remark 2.1(iii) is trivially satisfied when $F(x)=G(x)$ for all $x$. That this condition may hold even if $F(x) \neq G(x)$ is illustrated by the following example.

Example 2.3. Let $F(x)=1-\exp \left[-\lambda_{1} x\right], x \geq 0$, and $G(x)=1-\exp \left[-\lambda_{2} x\right], x \geq 0$, where $\lambda_{1} \geq \lambda_{2}>0$. Consider, for $x \geq 0$,

$$
\frac{\tilde{r}_{G}(x)}{\tilde{r}_{F}(x)}=\frac{\lambda_{2}}{\lambda_{1}} m(x),
$$

where

$$
m(x)=\frac{\exp \left[\lambda_{1} x\right]-1}{\exp \left[\lambda_{2} x\right]-1}
$$


Clearly,

$$
\begin{aligned}
m^{\prime}(x) & =\frac{\mathrm{d}}{\mathrm{d} x}(m(x)) \\
& =\frac{\lambda_{1} \exp \left[\lambda_{1} x\right]\left(\exp \left[\lambda_{2} x\right]-1\right)-\lambda_{2} \exp \left[\lambda_{2} x\right]\left(\exp \left[\lambda_{1} x\right]-1\right)}{\left(\exp \left[\lambda_{2} x\right]-1\right)^{2}}, \quad x \geq 0 .
\end{aligned}
$$

Let $\psi_{1}(x)=\exp \left[\lambda_{2} x\right]-1, x \geq 0$, and $\psi_{2}(x)=\exp \left[\lambda_{1} x\right]-1, x \geq 0$. Then

$$
\begin{array}{ll}
\psi_{1}^{\prime}(x)=\frac{\mathrm{d}}{\mathrm{d} x}\left(\psi_{1}(x)\right)=\lambda_{2} \exp \left[\lambda_{2} x\right], & x \geq 0, \\
\psi_{2}^{\prime}(x)=\frac{\mathrm{d}}{\mathrm{d} x}\left(\psi_{2}(x)\right)=\lambda_{1} \exp \left[\lambda_{1} x\right], & x \geq 0 .
\end{array}
$$

Applying Cauchy's mean value theorem to functions $\psi_{1}(\cdot)$ and $\psi_{2}(\cdot)$ on the interval $[0, x]$, we conclude that there exists a $\xi \in(0, x)$ such that

$$
\begin{gathered}
\frac{\psi_{1}(x)-\psi_{1}(0)}{\psi_{2}(x)-\psi_{2}(0)}=\frac{\psi_{1}^{\prime}(\xi)}{\psi_{2}^{\prime}(\xi)}, \\
\text { i.e. } \frac{\exp \left[\lambda_{2} x\right]-1}{\exp \left[\lambda_{1} x\right]-1}=\frac{\lambda_{2} \exp \left[\lambda_{2} \xi\right]}{\lambda_{1} \exp \left[\lambda_{1} \xi\right]} \geq \frac{\lambda_{2} \exp \left[\lambda_{2} x\right]}{\lambda_{1} \exp \left[\lambda_{1} x\right]}, \quad x \geq 0,
\end{gathered}
$$

or, equivalently,

$$
\lambda_{1} \exp \left[\lambda_{1} x\right]\left(\exp \left[\lambda_{2} x\right]-1\right)-\lambda_{2} \exp \left[\lambda_{2} x\right]\left(\exp \left[\lambda_{1} x\right]-1\right) \geq 0, \quad x \geq 0,
$$

which implies that $m^{\prime}(x) \geq 0$ for all $x \in \mathbb{R}_{+}$. Hence, $\tilde{r}_{G}(x) / \tilde{r}_{F}(x)$ is an increasing function of $x$ on $\mathbb{R}_{+}$.

Since the reversed failure rate ordering is also stronger than the usual stochastic ordering, it is of interest to know whether $T_{s}(\boldsymbol{k}) \leq_{\mathrm{rfr}} T_{S}\left(\boldsymbol{k}_{\mathbf{0}}\right)$ for all $\boldsymbol{k}, \boldsymbol{k}_{\mathbf{0}} \in \mathbb{K}$ such that $\boldsymbol{k}_{\mathbf{0}} \leq_{\mathrm{m}} \boldsymbol{k}$. The following example shows that this is not true if $n \geq 3$, even when $F \equiv G$. However, as proved in Theorem 2.2, the result is true for $n=2$ when $F \equiv G$.

Example 2.4. Consider the system $S_{\boldsymbol{k}}$ with $n=3, \boldsymbol{k}_{\mathbf{0}}=(4,8,8), \boldsymbol{k}_{\mathbf{1}}=(4,5,11)$, and $F \equiv G$. Clearly, $\boldsymbol{k}_{\mathbf{0}} \leq_{\mathrm{m}} \boldsymbol{k}_{\mathbf{1}}$. From (2.3), the reversed failure rate function of $T_{s}(\boldsymbol{k}), \boldsymbol{k} \in \mathbb{K}$, is given by

$$
\tilde{r}_{s, \boldsymbol{k}}(x)=\tilde{r}_{F}(x) \varphi_{\boldsymbol{k}}(F(x)),
$$

where, for $0<p<1$ and $\boldsymbol{k} \in \mathbb{K}$,

$$
\begin{aligned}
\varphi_{\boldsymbol{k}}(p)= & \frac{1}{1-\left(1-p^{k_{1}+1}\right)\left(1-p^{k_{2}+1}\right)\left(1-p^{k_{3}+1}\right)} \\
\times & {\left[\left(k_{1}+1\right) p^{k_{1}+1}\left(1-p^{k_{2}+1}\right)\left(1-p^{k_{3}+1}\right)\right.} \\
& \quad+\left(k_{2}+1\right)\left(1-p^{k_{1}+1}\right) p^{k_{2}+1}\left(1-p^{k_{3}+1}\right) \\
& \left.\quad+\left(k_{3}+1\right)\left(1-p^{k_{1}+1}\right)\left(1-p^{k_{2}+1}\right) p^{k_{3}+1}\right] .
\end{aligned}
$$

A simple calculation yields

$$
\varphi_{(4,8,8)}(p)-\varphi_{(4,5,11)}(p) \approx \begin{cases}-0.166 & \text { for } p=0.3 \\ +0.602 & \text { for } p=0.7\end{cases}
$$

implying thereby that, although $\boldsymbol{k}_{\mathbf{0}}=(4,8,8) \leq_{\mathrm{m}}(4,5,11)=\boldsymbol{k}_{\mathbf{1}}, T_{s}\left(\boldsymbol{k}_{\mathbf{1}}\right) \leq_{\mathrm{rfr}} T_{s}\left(\boldsymbol{k}_{\mathbf{0}}\right)$ does not hold. 
The following lemma (see Marshall and Olkin (1979, Theorem A.4)) will be used to prove the next theorem.

Lemma 2.3. Let $I \subseteq \mathbb{R}$ be an open interval, and let $\phi: I^{n} \rightarrow \mathbb{R}$ have continuous first-order partial derivatives:

$$
\phi_{(i)}(\boldsymbol{x})=\frac{\partial}{\partial x_{i}} \phi(\boldsymbol{x}), \quad i=1, \ldots, n, \boldsymbol{x}=\left(x_{1}, \ldots, x_{n}\right) .
$$

Then, if $\phi(\cdot)$ is permutation symmetric, i.e. $\phi(\boldsymbol{x})=\phi(\boldsymbol{y})$, whenever $\boldsymbol{x} \in I^{n}$ and $\boldsymbol{y}$ is a permutation of $\boldsymbol{x}$,

(i) $\phi(\cdot)$ is Schur convex if and only if $\left(x_{1}-x_{2}\right)\left(\phi_{(1)}(\boldsymbol{x})-\phi_{(2)}(\boldsymbol{x})\right) \geq 0$ for all $\boldsymbol{x} \in I^{n}$; and

(ii) $\phi(\cdot)$ is Schur concave if and only if $\left(x_{1}-x_{2}\right)\left(\phi_{(1)}(\boldsymbol{x})-\phi_{(2)}(\boldsymbol{x})\right) \leq 0$ for all $\boldsymbol{x} \in I^{n}$.

Theorem 2.2. Let $F_{1} \equiv F_{2} \equiv F \equiv G$. Then $\tilde{r}_{s, k}(x)$ is a Schur-concave function of $\boldsymbol{k}$ on $\mathbb{R}_{+}^{2}$ for all $x \geq 0$, i.e. $\tilde{r}_{s, \boldsymbol{k}}(x) \leq \tilde{r}_{s, \boldsymbol{k}_{\mathbf{0}}(x)}$ for all $x \geq 0$, whenever $\boldsymbol{k}, \boldsymbol{k}_{\mathbf{0}} \in \mathbb{R}_{+}^{2}$ and $\boldsymbol{k}_{\mathbf{0}} \leq_{\mathrm{m}} \boldsymbol{k}$.

Proof. From (2.3) we have, for $\boldsymbol{k} \in \mathbb{R}_{+}^{2}$,

$$
\tilde{r}_{s, \boldsymbol{k}}(x)=\tilde{r}_{F}(x) \psi_{F(x)}\left(k_{1}, k_{2}\right),
$$

where, for $0<p<1$ and $\boldsymbol{k} \in \mathbb{R}_{+}^{2}$,

$$
\psi_{p}\left(k_{1}, k_{2}\right)=\frac{k_{1} p^{k_{1}}+k_{2} p^{k_{2}}-\left(k_{1}+k_{2}+1\right) p^{k_{1}+k_{2}+1}}{p^{k_{1}}+p^{k_{2}}-p^{k_{1}+k_{2}+1}}+1 .
$$

We have

$$
\Delta_{p}\left(k_{1}, k_{2}\right) \equiv\left(\frac{\partial \psi_{p}\left(k_{1}, k_{2}\right)}{\partial k_{1}}-\frac{\partial \psi_{p}\left(k_{1}, k_{2}\right)}{\partial k_{2}}\right)=\frac{\psi_{2}(p)-(\ln p) p^{k_{1}+k_{2}} \psi_{1}(p)}{\left(p^{k_{1}}+p^{k_{2}}-p^{k_{1}+k_{2}+1}\right)^{2}},
$$

where

$$
\psi_{1}(p)=\left(k_{1}+1\right) p^{k_{2}+1}-\left(k_{2}+1\right) p^{k_{1}+1}+2\left(k_{2}-k_{1}\right), \quad 0<p<1,
$$

and

$$
\psi_{2}(p)=p^{2 k_{1}}-p^{2 k_{2}}-p^{2 k_{1}+k_{2}+1}+p^{k_{1}+2 k_{2}+1}, \quad 0<p<1 .
$$

In view of Lemma 2.3 we need to show that $\Delta_{p}\left(k_{1}, k_{2}\right) \geq 0$ whenever $0<p<1$ and $0 \leq k_{1} \leq k_{2}$. For this, suppose that $0 \leq k_{1} \leq k_{2}$. Then

$$
\psi_{1}^{\prime}(p)=\frac{\mathrm{d}}{\mathrm{d} p} \psi_{1}(p)=\left(k_{1}+1\right)\left(k_{2}+1\right)\left(p^{k_{2}}-p^{k_{1}}\right) \leq 0 \quad \text { for all } 0<p<1 .
$$

Therefore,

$$
\psi_{1}(p) \geq \lim _{p \rightarrow 1} \psi_{1}(p)=k_{2}-k_{1} \geq 0 \quad \text { for all } 0<p<1 .
$$

On applying Cauchy's mean value theorem to functions $f(x)=x^{k_{2}+1}, x \geq 0$, and $g(x)=$ $x^{k_{1}+1}, x \geq 0$, on the interval $[p, 1]$, we have, for $0<p<1$,

$$
\begin{gathered}
\frac{1-p^{k_{2}+1}}{1-p^{k_{1}+1}}=\frac{k_{2}+1}{k_{1}+1} \xi^{k_{2}-k_{1}} \quad \text { for some } \xi \in(p, 1) \\
\geq p^{2\left(k_{2}-k_{1}\right)} . \\
\Longrightarrow \quad \psi_{2}(p) \geq 0 \text { for all } p \in(0,1) .
\end{gathered}
$$

Using (2.10) and (2.11) in (2.9), the result follows. 
Now we assume that $X_{n} \leq_{\text {st }} X_{n-1} \leq_{\text {st }} \cdots \leq_{\text {st }} X_{1}$, i.e. the lifetimes $X_{1}, \ldots, X_{n}$ are stochastically ordered. Let $\mathbb{K}_{R}=\left\{\boldsymbol{L}: \boldsymbol{L} \in \mathbb{K}\right.$ and $\left.l_{1} \geq l_{2} \geq \cdots \geq l_{k}\right\}$. Theorem 2.3, below, generalizes a result of Boland et al. (1988). We require the following characterization of Schurconcave functions, similar to the characterization of Schur-convex functions in Marshall and Olkin (1979, p. 55).

Lemma 2.4. Let $\mathbb{D}^{*}=\left\{\boldsymbol{y} \in \mathbb{R}_{+}^{n}: y_{1} \geq y_{2} \geq \cdots \geq y_{n}\right\}$, and let $\phi$ be a function such that $\phi: \mathbb{D}^{*} \rightarrow \mathbb{R}^{1}, \mathbb{D}^{*} \subseteq \mathbb{R}^{n}$. Then $\boldsymbol{x} \leq_{\mathrm{m}} \boldsymbol{y}$ on $\mathbb{D}^{*}$ implies that $\phi(\boldsymbol{x}) \geq \phi(\boldsymbol{y})$ if and only if

$$
\phi(\boldsymbol{x}) \equiv \phi\left(\tilde{x}_{1}, \tilde{x}_{2}-\tilde{x}_{1}, \ldots, \tilde{x}_{n}-\tilde{x}_{n-1}\right)
$$

is a decreasing function of $\tilde{x}_{k}, k=1,2, \ldots, n-1$, over the region where $\boldsymbol{x} \in \mathbb{D}^{*}$. Here

$$
\tilde{x}_{k}=\sum_{i=1}^{k} x_{i}, \quad k=1,2, \ldots, n .
$$

Theorem 2.3. Let $X_{n} \leq_{\mathrm{st}} X_{n-1} \leq_{\mathrm{st}} \cdots \leq_{\mathrm{st}} X_{1}$, let $\mathbb{D}=\left\{\boldsymbol{L} \in \mathbb{K}_{R}: l_{1} \geq \cdots \geq l_{n}\right\}$, and let $\boldsymbol{k}_{\mathbf{0}}$ and $\boldsymbol{k}_{\mathbf{1}}$ be elements of $\mathbb{D}$ such that $\boldsymbol{k}_{\mathbf{0}} \leq_{\mathrm{m}} \boldsymbol{k}_{\mathbf{1}}$. Then $\bar{F}_{s, \boldsymbol{k}_{\mathbf{0}}}(x) \geq \bar{F}_{s, \boldsymbol{k}_{\mathbf{1}}}(x)$ for all $x \in \mathbb{R}$.

Proof. Fix $\boldsymbol{x} \in \mathbb{R}$. For $\boldsymbol{k} \in \mathbb{D}$, define

$$
\tilde{k}_{j}=\sum_{i=1}^{j} k_{i}, \quad j=1,2, \ldots, k, \quad \text { and } \quad \tilde{k}_{0}=0 .
$$

From (2.1) we have

$$
\bar{F}_{s, \boldsymbol{k}}(x)=\prod_{j=1}^{n}\left(1-F_{j}(x) G^{k_{j}}(x)\right)=\prod_{j=1}^{n}\left(1-F_{j}(x) G^{\tilde{k}_{j}-\tilde{k}_{j-1}}(x)\right), \quad x \in \mathbb{R} .
$$

Then, for $i=1,2, \ldots, k-1$,

$$
\begin{aligned}
\frac{\partial}{\partial \tilde{k}_{i}} \bar{F}_{s, \boldsymbol{k}}(x)= & (-\ln (G(x)))\left(\prod_{j=1}^{n}\left(1-F_{j}(x) G^{k_{j}}(x)\right)\right) \\
& \times\left(\frac{F_{i}(x) G^{k_{i}}(x)-F_{i+1}(x) G^{k_{i+1}}(x)}{\left(1-F_{i}(x) G^{k_{i}}(x)\right)\left(1-F_{i+1}(x) G^{k_{i+1}}(x)\right)}\right) \\
\leq & (-\ln (G(x)))\left(\prod_{j=1}^{n}\left(1-F_{j}(x) G^{k_{j}}(x)\right)\right) \\
& \times\left(\frac{F_{i+1}(x)\left(G^{k_{i}}(x)-G^{k_{i+1}}(x)\right)}{\left(1-F_{i}(x) G^{k_{i}}(x)\right)\left(1-F_{i+1}(x) G^{k_{i+1}}(x)\right)}\right) \\
\leq & 0,
\end{aligned}
$$

where the first inequality follows from the fact that $X_{i+1} \leq_{\mathrm{st}} X_{i}, i=1,2, \ldots, n-1$. Now, on using Lemma 2.4, the result follows.

Remark 2.2. (i) An interpretation of the above theorem is that if the stated conditions hold then a balanced allocation of spares yields higher reliability than the allocation which allows a larger number of spares for stronger components. 
(ii) When, in addition to the hypothesis of Theorem 2.3, the $X_{i}$ s are identically distributed for all $i=1, \ldots, n$, then, for every permutation $\boldsymbol{k}_{\beta}$ of $\boldsymbol{k}$,

$$
\bar{F}_{S, \boldsymbol{k}}(x)=\bar{F}_{S, \boldsymbol{k}_{\beta}}(x) \text { for all } x \in \mathbb{R} .
$$

Therefore, from Theorem 2.3, it follows that, for each $x \in \mathbb{R}, \bar{F}_{s, k}(x)$ is a Schur-concave function of $\boldsymbol{k}$ on $\mathbb{R}_{+}^{n}$.

The result stated in Remark 2.2(ii) was proved in Boland et al. (1988).

\section{Component redundancy versus system redundancy}

Consider a coherent system $\phi$ consisting of $n$ components $C_{1}, \ldots, C_{n}$ having independent and identically distributed lifetimes $X_{1}, \ldots, X_{n}$, respectively. Let $\bar{F}(\cdot), F(\cdot)$, and $f(\cdot)$ denote the common survival (reliability) function, the distribution function, and the probability density function, respectively, of the lifetimes of components. Let $\tau(\boldsymbol{X}) \equiv \tau\left(X_{1}, \ldots, X_{n}\right)$ denote the lifetime of the coherent system $\phi$. Then, the survival function of the coherent system $\phi$ is given by

$$
\mathrm{P}(\tau(\boldsymbol{X})>x)=K(\bar{F}(x)), \quad x \in \mathbb{R},
$$

where $K:[0,1] \rightarrow[0,1]$ is an increasing function with $K(0)=0$ and $K(1)=1$. Consider $n$ active spares $R_{1}, \ldots, R_{n}$ having independent and identically distributed lifetimes $Y_{1}, \ldots, Y_{n}$, respectively, each with survival function $\bar{G}(\cdot)$, distribution function $G(\cdot)$, and probability density function $g(\cdot)$. Assume that $\left(X_{1}, \ldots, X_{n}\right)$ and $\left(Y_{1}, \ldots, Y_{n}\right)$ are statistically independent.

In component redundancy we allocate an active redundant spare $R_{i}$ to the component $C_{i}, i=$ $1, \ldots, n$. Then the resultant coherent system, denoted by $S_{C}$, has lifetime $\tau(\boldsymbol{X} \vee \boldsymbol{Y}) \equiv \tau\left(X_{1} \vee\right.$ $\left.Y_{1}, \ldots, X_{n} \vee X_{n}\right)$ and survival function

$$
\bar{F}_{C}(x)=K(1-(1-\bar{F}(x))(1-\bar{G}(x))), \quad x \in \mathbb{R} .
$$

In system redundancy we duplicate the coherent system $\phi$ with components $C_{1}, \ldots, C_{n}$ replaced by $R_{1}, \ldots, R_{n}$ and make it available as an active redundant spare to the coherent system $\phi$. The resultant coherent system, denoted by $S_{S}$, has lifetime $\tau(\boldsymbol{X}) \vee \tau(\boldsymbol{Y})$ and survival function

$$
\bar{F}_{S}(x)=1-(1-K(\bar{F}(x)))(1-K(\bar{G}(x))), \quad x \in \mathbb{R} .
$$

For general coherent systems, it is well known (see Barlow and Proschan (1975, pp. 8 and 23)) that the component redundancy is better than the system redundancy with respect to the usual stochastic ordering, i.e.

$$
\tau(\boldsymbol{X}) \vee \tau(\boldsymbol{Y}) \leq_{\mathrm{st}} \tau(\boldsymbol{X} \vee \boldsymbol{Y})
$$

It will be interesting to explore if the above principle holds under other stochastic orders. Boland and El-Neweihi (1995) considered a two-component parallel system with $f(x)=\mathrm{e}^{-x}, x \geq 0$, and $g(x)=2 \mathrm{e}^{-2 x}, x \geq 0$, and, by plotting a graph between failure rate functions of component level redundancy and system level redundancy on $\mathbb{R}_{+}$, they observed that it is not true that

$$
\tau(\boldsymbol{X}) \vee \tau(\boldsymbol{Y}) \leq_{\text {fr }} \tau(\boldsymbol{X} \vee \boldsymbol{Y})
$$

Let, for an $n$-component parallel system, the lifetimes of components and spares be exponentially distributed with unequal failure rates $\lambda_{1}$ and $\lambda_{2}$. Then, it is interesting to know whether (3.1) holds for $\lambda_{1}>\lambda_{2}$. The following example shows that (3.1) is not true for any $\lambda_{1} \neq \lambda_{2}$. 
Example 3.1. Let $\tau(\boldsymbol{X})=\wedge\left(X_{1}, \ldots, X_{n}\right), \tau(\boldsymbol{Y})=\wedge\left(Y_{1}, \ldots, Y_{n}\right), \bar{F}(x)=\exp \left[-\lambda_{1} x\right]$, $x \geq 0$, and $\bar{G}(x)=\exp \left[-\lambda_{2} x\right], x \geq 0$, for $\lambda_{1}>0, \lambda_{2}>0$, and $\lambda_{1} \neq \lambda_{2}$. Then, for $x \geq 0$,

$$
\bar{F}_{C}(x)=\mathrm{P}(\tau(\boldsymbol{X} \vee \boldsymbol{Y})>x)=\left(\exp \left[-\lambda_{1} x\right]+\exp \left[-\lambda_{2} x\right]-\exp \left[-\left(\lambda_{1}+\lambda_{2}\right) x\right]\right)^{n}
$$

and

$$
\bar{F}_{S}(x)=\mathrm{P}(\tau(\boldsymbol{X}) \vee \tau(\boldsymbol{Y})>x)=\exp \left[-n \lambda_{1} x\right]+\exp \left[-n \lambda_{2} x\right]-\exp \left[-n\left(\lambda_{1}+\lambda_{2}\right) x\right] .
$$

Consider

$$
m(x)=\frac{\bar{F}_{C}(x)}{\bar{F}_{S}(x)}=\frac{\left(\exp \left[\lambda_{1} x\right]+\exp \left[\lambda_{2} x\right]-1\right)^{n}}{\exp \left[n \lambda_{1} x\right]+\exp \left[n \lambda_{2} x\right]-1}, \quad x \geq 0 .
$$

Clearly, for $\lambda_{1} \neq \lambda_{2}$,

$$
\lim _{x \rightarrow 0} m(x)=1 \quad \text { and } \quad \lim _{x \rightarrow \infty} m(x)=1 .
$$

Therefore, if $F \neq G$ then, in general, we cannot have

$$
\tau(\boldsymbol{X}) \vee \tau(\boldsymbol{Y}) \leq_{\text {fr }} \tau(\boldsymbol{X} \vee \boldsymbol{Y})
$$

In this section we investigate whether the redundancy at the component level is better than the redundancy at the system level with respect to system performance characteristics, such as reversed failure rate ordering, and the likelihood ratio order. For coherent systems with nonmatching components and spares, the following theorem provides the condition under which the redundancy at the component level is better than the redundancy at the system level under the reversed failure rate ordering.

Note that $X_{1}, \ldots, X_{n}$ denote the independent and identically distributed lifetimes of $n$ components and $Y_{1}, \ldots, Y_{n}$ denote the independent and identically distributed lifetimes of $n$ spares. Furthermore, $\bar{F}(\cdot), F(\cdot), f(\cdot)$ and $\bar{G}(\cdot), G(\cdot), g(\cdot)$ denote the common survival function, the distribution function, and the probability density function of independent and identically distributed random variables $X_{1}, \ldots, X_{n}$ and $Y_{1}, \ldots, Y_{n}$, respectively.

Theorem 3.1. If, for fixed $y \in(0,1)$,

$$
\frac{K^{\prime}(1-(1-x) y)}{K^{\prime}(x)}
$$

is a decreasing function of $x$ on $(0,1)$ then

$$
\tau(\boldsymbol{X}) \vee \tau(\boldsymbol{Y}) \leq_{\mathrm{rfr}} \tau(\boldsymbol{X} \vee \boldsymbol{Y}),
$$

where $K^{\prime}(\cdot)$ denotes the derivative of $K(\cdot)$.

Proof. For $t \in \mathbb{R}_{+}$,

$$
F_{C}(t)=\mathrm{P}(\tau(\boldsymbol{X} \vee \boldsymbol{Y}) \leq t)=1-K(1-(1-\bar{F}(t))(1-\bar{G}(t)))
$$

and

$$
F_{S}(t)=\mathrm{P}(\tau(\boldsymbol{X}) \vee \tau(\boldsymbol{Y}) \leq t)=(1-K(\bar{F}(t)))(1-K(\bar{G}(t)))
$$

Define

$$
m(t)=\frac{F_{C}(t)}{F_{S}(t)}=\frac{1-K(1-(1-\bar{F}(t))(1-\bar{G}(t)))}{(1-K(\bar{F}(t)))(1-K(\bar{G}(t)))}, \quad t>0 .
$$


We need to show that $m(t)$ is an increasing function of $t$ on $(0, \infty)$, i.e.

$$
m^{\prime}(t)=\frac{\mathrm{d}}{\mathrm{d} t}(m(t)) \geq 0 \quad \text { for all } t>0 .
$$

We have, for $t>0$,

$$
\begin{aligned}
((1- & K(\bar{F}(t)))(1-K(\bar{G}(t))))^{2} m^{\prime}(t) \\
= & (1-K(\bar{F}(t)))(1-K(\bar{G}(t)))(f(t) G(t)+g(t) F(t)) K^{\prime}(1-(1-\bar{F}(t))(1-\bar{G}(t))) \\
& -(1-K(1-(1-\bar{F}(t))(1-\bar{G}(t)))) \\
& \quad \times\left[g(t)(1-K(\bar{F}(t))) K^{\prime}(\bar{G}(t))+f(t)(1-K(\bar{G}(t))) K^{\prime}(\bar{F}(t))\right] \\
= & f(t)(1-K(\bar{G}(t)))\left[G(t)(1-K(\bar{F}(t))) K^{\prime}(1-(1-\bar{F}(t))(1-\bar{G}(t)))\right. \\
& \left.-(1-K(1-(1-\bar{F}(t))(1-\bar{G}(t)))) K^{\prime}(\bar{F}(t))\right] \\
& +g(t)(1-K(\bar{F}(t)))\left[F(t)(1-K(\bar{G}(t))) K^{\prime}(1-(1-\bar{F}(t))(1-\bar{G}(t)))\right. \\
& \left.-(1-K(1-(1-\bar{F}(t))(1-\bar{G}(t)))) K^{\prime}(\bar{G}(t))\right] \\
= & f(t)(1-K(1-y)) \psi(x, y)+g(t)(1-K(x)) \psi(1-y, 1-x),
\end{aligned}
$$

where $x=\bar{F}(t), y=G(t)$, and, for $0<s<1$ and $0<t<1$,

$$
\psi(s, t)=t(1-K(s)) K^{\prime}(1-t(1-s))-(1-K(1-t(1-s))) K^{\prime}(s) .
$$

To show that $m^{\prime}(t) \geq 0$ for every $t>0$, it is enough to show that $\psi(s, t) \geq 0$ whenever $0<s \leq 1$ and $0<t \leq 1$. Fix $s, t \in(0,1)$. On applying Cauchy's mean value theorem to functions $\psi_{t}(x)=K(1-t(1-x))$ and $\psi_{t}^{*}(x)=K(x)$ on the interval $[s, 1]$ we conclude that

$$
\frac{\psi_{t}(1)-\psi_{t}(s)}{\psi_{t}^{*}(1)-\psi_{t}^{*}(s)}=\frac{\psi_{t}^{\prime}(\xi)}{\psi_{t}^{*^{\prime}}(\xi)} \quad \text { for some } \xi \in(s, 1] .
$$

Under the hypothesis of the theorem, we have, from (3.2),

$$
\frac{1-K(1-t(1-s))}{1-K(s)}=t \frac{K^{\prime}(1-t(1-\xi))}{K^{\prime}(\xi)} \leq t \frac{K^{\prime}(1-t(1-s))}{K^{\prime}(s)}
$$

i.e. $\psi(s, t) \geq 0$. Hence, the result follows.

Corollary 3.1. Let $r \in\{1,2, \ldots, n\}$. Then, for $r$-out-of-n systems,

$$
\tau(\boldsymbol{X}) \vee \tau(\boldsymbol{Y}) \leq_{\mathrm{rfr}} \tau(\boldsymbol{X} \vee \boldsymbol{Y})
$$

Proof. We have, for $0<x<1$,

$$
K(x)=\frac{n !}{(n-r) !(r-1) !} \int_{1-x}^{1} u^{n-r}(1-u)^{r-1} \mathrm{~d} u
$$

and

$$
K^{\prime}(x)=\frac{n !}{(n-r) !(r-1) !}(1-x)^{n-r} x^{r-1} .
$$

For $0<y<1$,

$$
\frac{K^{\prime}(1-(1-x) y)}{K^{\prime}(x)}=y^{n-r}\left(\frac{1-y}{x}+y\right)^{r-1}
$$

is a decreasing function of $x$ on $(0,1)$. Hence, the result follows from Theorem 3.1. 
For coherent systems with identical components and spares, the following theorem provides the condition under which the redundancy at the component level is better than the redundancy at the system level under the likelihood ratio order.

Theorem 3.2. Let $F \equiv G$. Then $\tau(\boldsymbol{X}) \vee \tau(\boldsymbol{Y}) \leq 1 \mathrm{l} \tau(\boldsymbol{X} \vee \boldsymbol{Y})$ if and only if

$$
\psi_{1}(p)=\frac{1-K(p)}{1-p} \frac{K^{\prime}(p)}{K^{\prime}(p(2-p))}
$$

is an increasing function of $p$ on $(0,1)$.

Proof. We have

$$
\bar{F}_{C}(x)=\mathrm{P}(\tau(\boldsymbol{X} \vee \boldsymbol{Y})>x)=K(\bar{F}(x)(2-\bar{F}(x))), \quad x \in \mathbb{R},
$$

and

$$
\bar{F}_{S}(x)=\mathrm{P}(\tau(\boldsymbol{X}) \vee \tau(\boldsymbol{Y})>x)=1-(1-K(\bar{F}(x)))^{2}, \quad x \in \mathbb{R} .
$$

Then $\tau(\boldsymbol{X}) \vee \tau(\boldsymbol{Y}) \leq_{\operatorname{lr}} \tau(\boldsymbol{X} \vee \boldsymbol{Y})$ if and only if

$$
\begin{aligned}
L(x) & =\frac{(\mathrm{d} / \mathrm{d} x)(K(\bar{F}(x)(2-\bar{F}(x))))}{(\mathrm{d} / \mathrm{d} x)\left(1-(1-K(\bar{F}(x)))^{2}\right)} \\
& =\frac{1-\bar{F}(x)}{1-K(\bar{F}(x))} \frac{K^{\prime}(\bar{F}(x)(2-\bar{F}(x)))}{K^{\prime}(\bar{F}(x))}
\end{aligned}
$$

is an increasing function of $x$ on $\mathbb{R}_{+}$. Equivalently, $\tau(\boldsymbol{X}) \vee \tau(\boldsymbol{Y}) \leq \operatorname{lr} \tau(\boldsymbol{X} \vee \boldsymbol{Y})$ if and only if

$$
\psi_{1}(p)=\frac{1-K(p)}{1-p} \frac{K^{\prime}(p)}{K^{\prime}(p(2-p))}
$$

is an increasing function of $p$ in $(0,1)$.

The following corollary, proved in Singh and Singh (1997b), follows from Theorem 3.2.

Corollary 3.2. Let $r \in\{1,2, \ldots, n\}$, and let $F \equiv G$. Then, for $r$-out-of- $n$ systems,

$$
\tau(\boldsymbol{X}) \vee \tau(\boldsymbol{Y}) \leq_{\operatorname{lr}} \tau(\boldsymbol{X} \vee \boldsymbol{Y})
$$

Proof. For $r$-out-of- $n$ systems,

$$
K(p)=\frac{n !}{(n-r) !(r-1) !} \int_{1-p}^{1} u^{n-r}(1-u)^{r-1} \mathrm{~d} u, \quad 0<p<1 .
$$

Therefore,

$$
\begin{aligned}
\psi_{1}(p) & =\frac{1-K(p)}{1-p} \frac{K^{\prime}(p)}{K^{\prime}(p(2-p))} \\
& =\frac{n !}{(n-r) !(r-1) !} \frac{1}{(1-p)^{n-r+1}(2-p)^{r-1}} \int_{0}^{1-p} u^{n-r}(1-u)^{r-1} \mathrm{~d} u \\
& =\frac{n !}{(n-r) !(r-1) !} \int_{0}^{1} t^{n-r}\left(\frac{1+t}{2-p}-t\right)^{r-1} \mathrm{~d} t, \quad 0<p<1 .
\end{aligned}
$$

Clearly, $\psi_{1}(p)$ is an increasing function of $p$ on $(0,1)$. 


\section{Acknowledgements}

The authors are thankful to the anonymous referee for his/her valuable comments. The third author would like to acknowledge the financial assistance from the C.S.I.R., India, for carrying out this research work.

\section{References}

Barlow, R. E. And Proschan, F. (1975). Statistical Theory of Reliability and Life Testing. Holt, Rinehart and Winston, New York.

Boland, P. J. AND El-NeweIHI, E. (1995). Component redundancy vs system redundancy in the hazard rate ordering. IEEE Trans. Reliab. 44, 614-619.

Boland, P. J., El-Neweihi, E. And Proschan, F. (1988). Active redundancy allocation in coherent systems. Prob. Eng. Inf. Sci. 2, 343-353.

Kalashnikov, V. V. And Rachev, S. T. (1986). Characterization of queueing models and their stability. Prob. Theory Math. Statist. 2, 37-53.

Marshall, A. W. And Olkin, I. (1979). Inequalities: Theory of Majorization and Its Applications. Academic Press, New York.

Pečarić, J. E., Proshan, F. And Tong, Y. L. (1992). Convex Functions, Partial Orderings and Statistical Applications. Academic Press, Boston, MA.

Sengupta, D. and Deshpande, J. V. (1994). Some results on the relative ageing of two life distributions. J. Appl. Prob. 31, 991-1003.

Shaked, M. And Shanthikumar, J. G. (1992). Optimal allocation of resources to nodes of parallel and series systems. Adv. Appl. Prob. 24, 894-914.

Shaked, M. And Shanthikumar, J. G. (2007). Stochastic Orders. Springer, New York.

SingH, H. AND SINGH, R. S. (1997a). Note: optimal allocation of resources to nodes of series systems with respect to failure-rate ordering. Naval Res. Logistics 44, 147-152.

Singh, H. AND Singh, R. S. (1997b). On allocation of spares at component level versus system level. J. Appl. Prob. 34, 283-287. 